\title{
The Genomics of Prostate Cancer: emerging understanding with technologic advances
}

\author{
Mark A Rubin ${ }^{1,2,3,4}$ and Francesca Demichelis ${ }^{1,5}$ \\ ${ }^{1}$ Institute for Precision Medicine, Weill Cornell Medical College-New York Presbyterian Hospital, New York, \\ NY, USA; ${ }^{2}$ Sandra and Edward Meyer Cancer Center at Weill Cornell Medical College, New York, NY, USA; \\ ${ }^{3}$ Department of Pathology and Laboratory Medicine, Weill Cornell Medical College, New York, NY, USA; \\ ${ }^{4}$ Department of Biomedical Research, University of Bern, Bern, Switzerland and ${ }^{5}$ Centre of Integrative Biology, \\ University of Trento, Trento, Italy
}

\begin{abstract}
With the advent of next-generation sequencing technologies and large whole-exome and genome studies in prostate and other cancers, our understanding of the landscape of genomic alterations has dramatically been refined. In additional to well-known alterations in genomic regions involving 8p, 8q, 10q23, common ETS translocations and androgen receptor amplifications, newer technology have uncovered recurrent mutations in SPOP, FOXA1, MED12, IDH and complex large scale genomic alterations (eg, chromoplexy). This review surveys the enhanced landscape of genomic alterations in clinically localized and advanced prostate cancer.

Modern Pathology (2018) 31, S1-S11; doi:10.1038/modpathol.2017.166
\end{abstract}

The genomics of prostate cancer (PCA) has been difficult to study compared with some other cancer types for a multitude of reasons, despite significant efforts since the early 1980s (Figure 1). First, the anatomic location of the prostate gland has initially made it challenging to obtain samples for research. This was largely overcome with the widespread acceptance of the nerve sparing radical prostatectomy. ${ }^{1}$ Second, unlike melanoma, lung and breast cancers, PCA does not lend itself to simple growth in culture. From the earliest attempts to grow PCA in vitro to develop short-term cultures or to develop karyotypes, it was clear that cells would not readily grow and most often undergo senescence or are overtaken by benign fibromuscular stromal cells. The effects have severely hampered PCA research limiting model systems for understanding the basic biology and genomics of the disease. Recent work in the development of PCA organoids has reignited interest in developing novel model systems for biologic and genomic studies that more closely reflect the PCA observed in patients. ${ }^{2}$ Third, it has been traditionally difficult to obtain samples from advanced disease. However, now improved care for men with advanced castrationresistant prostate cancer (CRPC) has led to longer

Correspondence: Professor MA Rubin, MD, Department for Biomedical Research, University of Bern, Murtenstrasse 35, Bern 3008, Switzerland.

E-mail: mark.rubin@dbmr.unibe.ch or Dr F Demichelis, PhD, Centre for Integrative Biology University of Trento, Via Sommarive 9, Trento 38123, Italy.

E-mail: f.demichelis@unitn.it

Received 29 July 2017; revised 10 October 2017; accepted 10 October 2017 survival. As a consequence, there is more acceptance for metastatic bone and soft tissue biopsies making CRPC samples available for genomic studies. ${ }^{3}$

The advent of high-throughput technologies including comparative genomic hybridization (CGH), fluorescence in situ hybridization (FISH), array CGH (aCGH), gene expression profiling and more recently the introduction of massively parallel genomic sequencing referred to as next-generation sequencing (NGS) has led to a deeper understanding of cancer genomics in general. The increased developments in the field of computational biology that have paralleled these advances have also led to widely available genomic data sets that have encouraged researchers from many fields to contribute to our understanding of genomics.

This review will provide an overview of the PCA genomic landscape with an emphasis on the cardinal mutations and alterations observed to be consistently seen in PCA (Figure 1), both hormone naive localized PCA and CRPC.

\section{Cardinal PCA mutations}

Cancer is a genetic disease with two major classes of significant mutations: inactivating mutations (in tumor suppressors) and activating mutations (in oncogenes). Inactivation often comes from structural rearrangements involving loss of genomic DNA resulting in deletions (large or focal) or rearrangements. In both cases, a gene or groups of genes are disrupted. These events can be either mono(heterozygous) or bi-allelic (homozygous). Activation can occur through amplification, point mutation or 


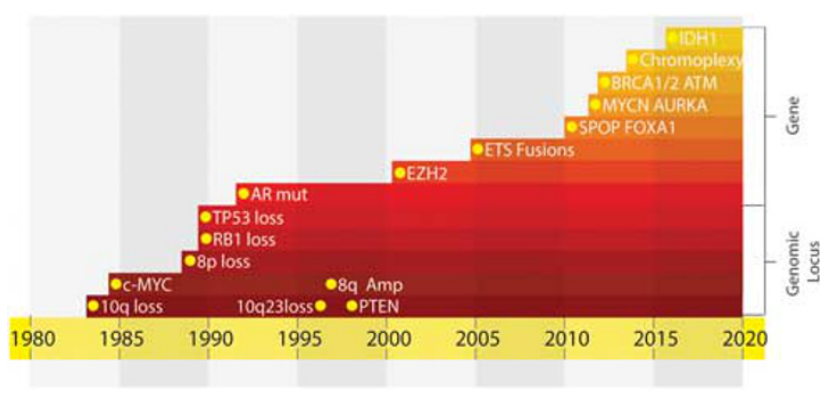

Figure 1 Timeline of cardinal genomic lesions in PCA.

structural rearrangements leading to gene fusions. The cardinal recurrent alterations in PCA include 8p, 8q (C-MYC locus), 10p (PTEN locus), 17q (TP53 locus) and androgen receptor (AR).

\section{0q Loss is Common in PCA}

Atkin and Baker ${ }^{4}$ were the first to describe in human PCA samples chromosome 10 deletion at 10q24. A prior study on the PCA cell line LNCaP had reported 10q24 loss. ${ }^{5}$ Later reports confirmed this 10q24 locus as a site for current deletion in PCA. ${ }^{6-8}$ Mitelman's group demonstrated 10q loss (10q24) occurred in 5/33 (15\%) PCA cases studied.

\section{TP53 (Cellular Tumor Antigen p53)}

Studies based on the restriction fragment length polymorphism (RFLP) approach helped discovery and define the role of TP53 as a tumor-suppressor altered in multiple cancer types including lung, colon, breast, bladder and brain cancers. ${ }^{9-11}$ Using this approach, Carter et $a 1^{12}$ defined recurrent $\mathrm{LOH}$ on a number of loci in PCA including the 17p locus harboring TP53. Isaacs et $a l^{13}$ demonstrated for the first time the presence of p53 mutations in cell lines (eg, PC-3 and DU145) and two primary human PCA samples. They also demonstrated the functional role of wild-type (WT) p53 has as a tumor suppressor. This study helped define p53 as a tumor suppressor in PCA. Subsequent studies demonstrated that in larger numbers of patient samples, p53 mutations accumulated with disease progression. ${ }^{14,15}$ Specifically, Navone et al ${ }^{15}$ in 1993 detected p53 mutations in 23 of 53 AR (43\%) 'independent' PCA (referred to today as CRPC) in contrast to no mutations seen in 44 AR-dependent PCA samples. Enrichment for p53 mutations with PCA disease progression has been confirmed in numerous studies as a consistent event. In 2016, Robinson et al ${ }^{3}$ reported 53\% mutations in the Stand Up 2 Cancer/ Prostate Cancer Foundation CRPC500 study cohort (referred hereon as CRPC500 cohort).

\section{RB1 (Retinoblastoma-Associated Protein)}

RB1 was originally localized to 13q14.1 by cytogenetic analysis from patients with retinoblastoma ${ }^{16}$ and cloned by Friend et al in 1986. ${ }^{17}$ In 1990, Bookstein et $a l^{18}$ reported RB1 loss in the DU145 PCA cell line and functionally associated loss with increased tumorigenicity, which could be reversed by reintroduction of RB1. Early studies suggested RB1 loss ranging from around 30 to $60 \%$ using RFLP analysis for the 13q RB1 locus. ${ }^{19-21}$ In the CRPC500 cohort, RB1 is reported lost in $21 \%$ of cases. ${ }^{3}$

\section{8p Loss}

The first report of $8 \mathrm{p}$ loss in PCA was by Konig et al ${ }^{22}$ in 1989 on the LNCaP cell line. Numerous groups confirmed this observation in primary and advanced PCA. ${ }^{20,23-34}$ Although candidate tumor-suppressor genes had been proposed in this region, ${ }^{35}$ it was not until 1997 that $\mathrm{He}$ et $a l^{36}$ identified in a prostatespecific gene, NKX3.1, which was homologous to the Drosophila NK homeobox gene family. NKX3.1 is expressed at high levels in normal prostate and is androgen sensitive as they determined by androgen stimulation of LNCaP cell lines. They mapped NKX3.1 to chromosome band 8 p21, a region that was previously noted to undergo loss. He et al proposed a potential tumor-suppressor role for NKX3.1.

\section{Androgen Receptor}

Mutations have been long known to exist in the AR. AR mutations occur and result in a germline disorder called androgen insensitivity syndrome, an $\mathrm{X}$ chromosome-linked inherited disorder (reviewed in Hughes et $a l^{37}$ and Shukla et $a l^{38}$ ). Mutations in the ligand-binding domain of the AR receptor were first observed in an androgen-responsive PCA cell line, LNCaP. ${ }^{39}$ Newmark et $a l^{40}$ reported the first AR mutations associated with primary PCA. Frequent AR mutations were observed in CRPC (50\%) demonstrating for the first time that $\mathrm{AR}$ resistance via mutation occurs with AR-targeted therapy. ${ }^{41}$ Another mechanism for AR resistance can be explained by AR gene amplification. aCGH and FISH technology helped define 4-fold to over 20-fold AR amplifications in hormone-treated PCA patients but not in untreated hormone naive PCA. ${ }^{42}$ With the development of tissue microarray (TMA) technology, larger numbers of clinical samples could be detected on a single slide. Using TMAs, Bubendorf et al ${ }^{43}$ queried the AR status on 371 PCA samples by FISH. AR was determined to be amplified in $23 \%$ of the 47 CRPC cases in contrast to 2 of $205(1 \%)$ of the primary hormone naive PCA cases. In more recent studies using NGS, the AR aberration frequencies show the same patterns. In the TCGA study of 333 hormone naive PCA, no AR mutations were detected. ${ }^{44}$ In studies where tumors were evaluated after androgen deprivation therapy (ADT), AR mutations and amplification frequencies were in the range of the initial reports. ${ }^{3,45-48}$ Other mechanism of AR 
resistance have been proposed including v7 AR splice variants ${ }^{49}$ and lineage plasticity to AR indifferent CRPC. ${ }^{45,50}$

\section{$10 q 23$ (PTEN)}

The distal region of $10 \mathrm{q}$ is lost in a number of common cancers such as glioblastoma and breast cancer. Early studies using RFLP assays located the loss at 10q24, but a series of articles in the 1990s targeted 10q23.1 as a potential site for a tumorsuppressor gene. In 1990, Carter et $a l^{12}$ reported $10 \mathrm{q}$ loss in around 30\% of localized PCA. In 1995, Gray et $a l^{51}$ suggested the critical area for a potential tumor suppressor was in 10q23-24 lost in $62 \%$ of the 37 PCA cases they examined. In 1996, Ittmann was the first to propose 10q23.1 demonstrating increased loss in advanced PCA. He also suggested that prior studies using aCGH approaches may have missed the 10q23.1 region as deletions in some cases were small.

Mapping of multiple cancers including brain, breast and prostate, Ramon Parson's group pinpointed a minimal area of genomic deletion at 10q23.1 leading to the cloning of the candidate tumor-suppressor gene phosphatase and tensin homolog deleted on chromosome ten (PTEN). ${ }^{52}$ Mutations were detected in brain and breast cancer. All PCA cell lines tested demonstrated either mutations (ie, LNCaP and DU145) or homozygous deletions (ie, NCI-H660 and PC-3). Since the initial work, few inactivating PTEN mutations have been detected in PCA. ${ }^{53}$ PTEN has a critical role in regulating the PI3K-AKT pathway such that loss leads to downstream activation.

\section{MYC Amplification (8q24)}

C-Myc is a transcription factor with a wide range of functions including modulation of protein synthesis, cell cycle and metabolism. C-myc, the protein encoded by the MYC oncogene on 8q24, was observed in 1986 as overexpressed in human primary PCA. Flemming et al $1^{54}$ reported c-myc overexpression at the transcript level using Northern blot technology. In 1997, Jenkins et al ${ }^{55}$ conducted the first extensive study using FISH at $8 \mathrm{q} 24$ to demonstrate gene amplification of MYC. Amplification of MYC was observed in 25\% of the clinically localized PCA tumors but in $46 \%$ of the advanced PCA samples examined, suggesting that MYC amplification corresponds to disease progression. Interestingly, they also observed that in the localized samples, MYC amplification was often only amplified in a subset of the tumor cells in the lesion consistent with genomic heterogeneity. MYC amplification is one of the genomic events that appears to be significantly different in CRPC vs primary PCA.

The importance of co-occurring molecular alterations is well illustrated by the amplification of MYC and activation of the PI3K-pathway. Clegg et a ${ }^{56}$ observed that there is a statistically significant association between PI3K pathway alterations (ie, PTEN, PI3CA, AKT1, AKT2 and AKT3) and MYC amplification with $27 \%$ and $70 \%$ co-occurrence in localized and metastatic PCA, respectively. To determine the potential impact of these cooccurring genomic alterations, they developed a series of genetically engineered mouse models (GEMs) to explore the relationships of the individual and co-occurring alterations. Using mice with either PTEN loss or AKT overexpression and crossing them, respectively, with high MYC overexpressing mice 57 in a prostate conditional context, they demonstrated that the addition of MYC leads to an acceleration of PIN and adenocarcinoma. Interestingly, whereas RAD001, a rapamycin analog, can inhibit the formation of PIN in prostate conditional AKT-activated GEMs, RAD001 did not abrogate the development of PIN in mice expressing both AKT and MYC. This suggests that MYC acts in a manner that is independent from mammalian target of rapamycin C1 (mTORC1) activation. These important studies begin to reveal the complexity of cooccurring genomic alterations in cancer, the additional challenges to therapeutic strategies and the need to better understand them through model systems.

\section{SCNAs define PCA risk progression}

CGH identified somatic copy number alterations (SCNAs) in a high percentage $(\sim 75 \%)$ of localized PCA. Losses were found to be five times more common than gains and most often involved $8 \mathrm{p}$ (32\%), 13q (32\%), 6q (22\%), 16q (19\%), 18q (19\%) and $9 \mathrm{p}(16 \%)$. These early genome-wide studies also suggested that the pattern of copy number alterations changes with disease progression. For example, gains of $7,8 q$ and $X$ were more often observed in the CRPC state. ${ }^{23}$ La Pointe et $a l^{58}$ proposed a subclassification of PCA risk based on aCGH data with three potential risk groups. More recently, refined maps of SCNAs have demonstrated significant association with Gleason grade prognostic groups ${ }^{59}$ and overall SCNAs as a measure of PCA relapse. ${ }^{60}$ In recent work in populations of CRPC, distinct SCNAs in AR and MYC have been confirmed from prior studies. ${ }^{3}$ Therefore, the overall burden and specific genomic alterations may potentially become useful PCA biomarkers.

\section{ETS rearrangements in PCA}

There is an emerging view of PCA suggesting a high degree of molecular complexity involving common recurrent gene fusions, ${ }^{61}$ large complex genomic rearrangements ${ }^{62}$ and common recurrent mutations. $^{44,62-64}$ This heterogeneity might be best viewed as a collection of more homogenous subgroups defined by SCNAs, mutations and 
rearrangements. The most common gene fusion in PCA is the TMPRSS2:ERG fusion. ${ }^{61}$ The discovery of the ETS family transcription factor gene fusions in 2005 dramatically changed the view that gene fusions predominately occurred in sarcomas and hematopoietic malignancies. ${ }^{61}$ Cancer outlier profile analysis on existing PCA expression microarray experiments nominated two consistently high-scoring, and mutually exclusive candidates across 50\% of PCA samples, which were members of the ETS family of transcription factors, ERG and ETV1. Further experiments revealed fusions of the 5'untranslated region of TMPRSS2 (21q22.3) with the ETS transcription factor family members, either ERG (21q22.2), ETV1 (7p21.2) ${ }^{61}$ or ETV4, ${ }^{65}$ suggesting a novel mechanism for overexpression of the ETS genes in PCA. In addition to TMPRSS2, three other androgen-responsive 5' partners SLC45A3, ${ }^{66,67} \mathrm{HER}$ $P U D 1^{68}$ and NDRG1 ${ }^{69}$ have been found to fuse with ERG.

Recent advances in next-generation transcriptome sequencing facilitated the discovery of RAF kinase gene fusions, SLC45A3-BRAF, ESRP1-RAF1 and RAF1-ESRP1 in advanced PCA. ${ }^{70}$ Although rare, detected in $\sim 1-2 \%$ of PCAs, RAF kinase fusions represent the first 'driver' fusion in PCA that do not involve an ETS family member.

\section{Emerging Understanding of PCA Genomic Complexity}

Genomic rearrangements appear to be nonrandom, locus specific and depend, in part, on the proximity of chromosomal regions in the nucleus. ${ }^{71}$ Moreover, there is a mounting evidence suggesting that transcription factors are associated with DNA doublestrand breaks, thus predisposing transcribed regions to genomic rearrangements. For example, both androgen and estrogen signaling recruits the enzyme topoisomerase $2 \mathrm{~B}$ (TOP2B) to target gene promoters, which creates DNA double-strand breaks and facilitates transcription. ${ }^{72,73}$ The AR and TOP2B are coexpressed in human PCA precursor lesions in which TMPRSS2:ERG rearrangements are known to occur, suggesting a critical role of TOP2B in the recurrent ETS rearrangements. Three recent studies have also shown that androgen signaling promotes TMPRSS2: ERG fusion formation, ${ }^{74-76}$ in part, by recruiting DNA break-inducing enzymes (eg, activation of induced cytidine deaminase) to translocation breakpoint sites. ${ }^{75}$ More recently, Berger et al ${ }^{62}$ demonstrated that rearrangement breakpoints were enriched near open chromatin, AR and ERG DNAbinding sites in the setting of the ETS gene fusion TMPRSS2:ERG, but inversely correlated with these regions in tumors lacking ETS fusions. Hence, transcription factors can contribute to the formation of genomic rearrangements by facilitating the juxtaposition of chromosomal loci and recruiting enzymatic machinery involved in DNA breaks to these target loci.
In summary, ETS fusions are the most common recurrent genetic mutation identified in PCA. Although a number of ETS and non-ETS family members have been observed to be fused with TMPRSS2 or other 5' partners, the vast majority of fusions involve TMPRSS2:ERG. This fusion can be studied in large numbers, as it was identified in approximately $45 \%$ of all prostate-specific antigen (PSA) screened PCA.

\section{Molecular landscape of PCA in the NGS era}

Comprehensive NGS studies characterizing the PCA genomic landscape have identified a few highly recurrent somatically mutated genes (including SPOP, TP53, PTEN and FOXA1, all <15\%), with recurrent broad copy number alterations (CNAs; ie, $8 p$ loss and $8 \mathrm{q}$ gain), but relatively few focal and/or high-level CNAs (most commonly focal PTEN, TP53 and $R B 1$ losses). The recent TCGA publication of 333 PCA genomes, transcriptomes and methylomes solidifies the idea of PCA molecular subclasses. ${ }^{77}$ One major advantage of NGS generated tumor data sets is the possibility to computationally assess tumor cellularity (fraction of tumor cells in cells constellation whose DNA was analyzed, also referred to as tumor purity) by exploiting single base resolution data and to infer tumor aneuploidy, as accomplished by various tools including ABSOLUTE ${ }^{78}$ and CLONET. ${ }^{79}$ In addition, NGS data make it possible to assess the clonality of each single somatic lesion (fraction of tumor cells that harbor a specific somatic lesion) allowing for the definition of the first primary PCA evolution chart $^{63}$ and of phylogenic trees to trace tumor history in one patient when for instance multiple metastatic biopsies are sequenced. ${ }^{48,80}$ Altogether these computational assessments from large collection of prostatectomies samples characterized through whole-exome sequencing (WES) or whole-genome sequencing (WGS) deepened our recognition of earlier vs later events in prostate carcinogenesis.

\section{Insights into Genomic Complexity}

With the advent of WGS, insights into global genomic rearrangement are possible. Berger et al ${ }^{62}$ published the first seven whole genomes revealing structurally complex rearrangements. ${ }^{62}$ The associations were significantly enriched for disruption of tumor-suppressor genes. There was also an observation that the rearrangements in the ETS rearrangement cancers tended to occur in the proximity of transcriptionally open chromatin marks suggesting that subclasses of PCA may act differently based on early genomic alterations. In a significantly larger WGS study, Baca et $a^{63}$ extended these observations based on 57 PCAs. Using statistical arguments and 
simulations, they found that the many complex rearrangements likely originated within a singlecell cycle, providing a proliferative advantage to a cancerous cell causing multiple oncogenic events. This was termed 'chromoplexy' to refer to these distinctive genomic restructuring events. They demonstrated that this process is distinct from the phenomenon of chromothripsis. ${ }^{81}$ Chromoplexy is active across the majority of PCAs and in several non-prostate tumor lineages. Similarly, in a recent publication Fraser and colleagues ${ }^{82}$ describe results from a large WGS effort on localized, intermediaterisk PCA that includes specific single-nucleotide variants and rearrangement signatures with DNA footprints associated with ageing and clustered mutation phenomenon called kataegis. This large WGS study demonstrates a high burden of complex rearrangements, including samples with evidence of chromothripsis (chromosome shattering). Although not reported, their study also demonstrated chromoplexy (chains of balanced rearrangements) altogether suggesting highly altered 3D structure of PCA cell genome (personal communication).

Another observation from WGS analysis revealed that while the ETS fusion cases demonstrated a high frequency of intergenic gene rearrangements, a second group of around $10 \%$ of PCA demonstrated high frequencies of intragenic rearrangements. This later class is best defined as harboring SPOP mutations and CHD1 loss. ${ }^{83}$ This recent work had demonstrated that SPOP mutations are associated with a homology repair defect phenotype.

\section{SPOP Mutations Define a Distinct Molecular Subclass of PCA}

Recurrent missense mutations in SPOP are the most common point mutations in primary PCA, occurring in about $10 \%$ of both clinically localized and metastatic CRPC. ${ }^{3,44,62,84,85}$ The most common hot spot SPOP mutations occur at F133, Y87, F102 and

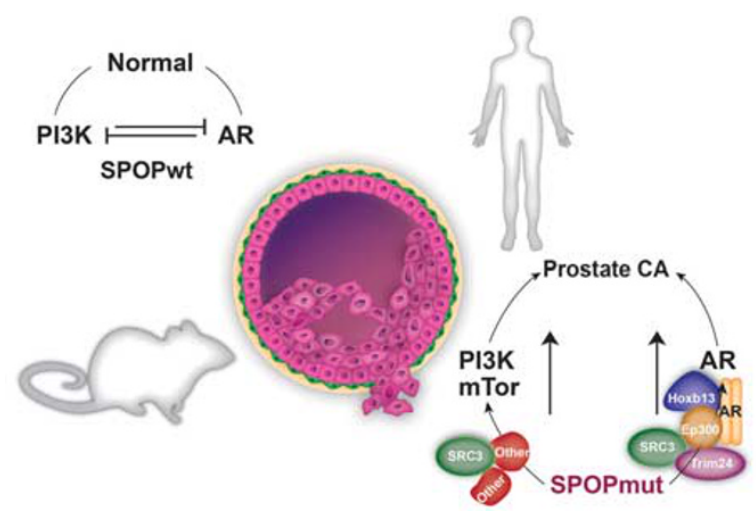

Figure 2 Schematic of human SPOP mutation potential to drive prostate tumorigenesis. Mouse model work shows that human SPOP mutation can drive prostate tumorigenesis through coordinate deregulation of PI3K/mTOR and AR pathways.
W131 and often co-occur with specific genomic features including deletions at 5q21, 6q15 and 2q21. ${ }^{83,84}$ Molecularly, human PCA can be classified into those harboring rearrangements in ETS transcription factors (eg, TMPRSS2-ERG) and those lacking ETS rearrangements. SPOP mutant PCA also defines characteristic genomic rearrangements, gene expression profiles and methylation patterns. ${ }^{44,83-85}$ SPOP mutations occur early in the natural history of PCA solely as heterozygous missense mutations with dominant negative, selective loss of function toward the remaining WT allele. ${ }^{63,79,83,86}$

SPOP encodes the substrate recognition component of a CUL3-based E3 ubiquitin ligase, and PCAderived SPOP mutants appear to act as dominant negatives with selective loss-of-function. ${ }^{83}$ Known substrates of $S P O P$ are numerous, and the specific substrates that are deregulated by $S P O P$ mutations are starting to be defined. These include the chromatin-associated oncogene DEK, ${ }^{86}$ the oncogenic co-activator TRIM $24^{86,87}$ and the AR itself. 88,89

Initial models have established the role of SPOP mutation as a driver of prostate neoplasia in vivo, and studies exploring the downstream effects of $S P O P$ mutations have largely relied on overexpression of mutant $S P O P$ protein in cell lines with alterations outside the genetic context of SPOP mutant PCA. ${ }^{86,89-92}$

Blattner et $a{ }^{93}$ reported the development of the first conditional mouse model showing that SPOP mutation drives prostate tumorigenesis in vivo. Using in vitro models derived from these mice, they demonstrated that mutant SPOP activated both PI3K/ mTOR signaling and AR signaling, effectively uncoupling the normal negative feedback between these two pathways. Together, these findings show that SPOP mutation drives prostate neoplasia in vivo through deregulation of the PI3K/mTOR and AR pathways, and underscore the critical role of these two signaling pathways across molecular subtypes of human PCA (Figure 2). More recent work has also suggested a genetic predisposition to this distinct subclass of PCA and this may be modulated by AR signaling and DNA repair alterations. ${ }^{94}$

\section{Genomic alterations and resistance}

It has been long observed that AR mutations are only seen in the setting of treated PCA. Recent genomics analysis has provided a deeper understanding as to the extent and repertoire of alterations associated with resistance.

Multiple studies have now assessed the molecular landscape of lethal CRPC (predominantly from rapid autopsy samples). ${ }^{47,95-99}$ WES and targeted NGS studies have demonstrated that $A R$ is the most recurrent alteration (approximately 50\%) in CPRC but not in untreated PCA (from unrelated patients or previous studies), consistent with the known role of 
$A R$ in mediating CRPC progression. ${ }^{100-106}$ Nevertheless, numerous genes are altered more frequently in CRPC vs untreated PCA (ie,TP53 and PTEN), and additional potential low-frequency driving alterations are observed more frequently in lethal CRPC than untreated PCA (ie, deleterious APC alterations in $20 \%$ of CRPC and $3-5 \%$ of untreated PCA from TCGA and our previous untreated PCA studies). ${ }^{47,63,64,106}$ Similarly, single gene, functional and profiling studies have identified pathways that may mediate progression to CRPC along with AR pathway deregulation, such as the WNT/APC/ CTNNB1 or PI3K pathways, ${ }^{47,106-111}$ however, it is unclear if these alterations were present before ADT, mediated progression to CRPC or acquired after CRPC progression.

\section{AR Indifferent PCA: Another Mechanism for Resistance}

Treatment-related neuroendocrine prostate cancers (NEPC) are clonally derived from a castrationresistant adenocarcinoma precursor (see recent review Rickman et $a l^{112}$ ). Although they retain PCA genomic alterations, NEPC tend to lose expression of common prostate markers and are often treated with regimens similar to small cell lung cancer. There is a spectrum of disease within CRPC, with small cell carcinoma being the extreme and mixed and intermediate atypical phenotypes also observed which can retain AR expression and harbor overlapping molecular features. Clinical features associated with platinum sensitivity (termed 'anaplastic' or 'aggressive variant' PCA) have been defined for patient selection in Phase 2 chemotherapy studies and share pathologic and molecular features of NEPC. The cell plasticity associated with NEPC is associated with decreased or absent expression of the AR and downstream androgen-regulated genes such as PSA. Moreover, data from pre-clinical models also favor a trans-differentiation model of treatment-related NEPC. For example, adenocarcinoma cells (eg, LNCaP) display a neuroendocrine phenotype similar to the few established NEPC cell lines that have been described (eg, NCI-H660) $)^{50,113,114}$ following exposure to a variety of stimuli (eg, androgen deprivation ${ }^{115}$ or treatment with cAMP, ${ }^{116} \mathrm{IL}^{1} \mathrm{6}^{117}$ or fractionated ionizing radiation). ${ }^{118}$ Similar trans-differentiation results have been observed in vivo. For example, a well-characterized patient-derived prostate adenocarcinoma xenografts (LTL331) implanted into the subrenal capsule of mice develops small cell NEPC following castration (LTL331R ${ }^{119}$ and which phenotypically resembled other lines that were generated from bone fide NEPC tumor tissue (eg, LTL352, LTL370, ${ }^{119}$ LuCAP-49, ${ }^{120}$ UCRU-PR-2 ${ }^{121}$ and WISHPC2). ${ }^{122}$ Genomic profiling at various time-points during trans-differentiation suggests clonal evolution from the adenocarcinoma LTL331 to the NEPC LTL331R tumor. ${ }^{119}$
Activation of Myc family oncogenes commonly occur across poorly differentiated neuroendocrine carcinomas and in small cell lung cancer, up to $20 \%$ harbor genomic amplification of N-myc, C-Myc and L-Myc, or a recurrent fusion involving MYCL1 (9\%). ${ }^{123-126}$ N-Myc amplification occurs in highrisk neuroblastoma and a subset of NEPC and SCLC. $\mathrm{N}-\mathrm{Myc}$ has also been shown to be amplified and overexpressed in NEPC and can drive the NEPC phenotype. ${ }^{50,127,128}$ Recently, Dardenne et $a 1^{129}$ showed using a variety of pre-clinical models including isogenic cell lines, xenografts, GEMM and mouse tumor organoids cultures, that N-Myc overexpression is associated with highly proliferative, invasive PCA with pathologic features of NEPC. Dardenne et al further showed that the N-Myc interacts and cooperates with enhancer of zeste homolog 2 (EZH2) to drive NEPC transcriptional programs including an abrogation of AR signaling, enhanced AKT and EMT signaling, repression of polycomb repressive complex 2 (PRC2) target genes and expression of neuroendocrine markers. Lee et $a l^{127}$ used a forward engineering approach of benign human prostate cell that overexpress N-Myc in the context of myristoylated AKT and provided compelling data showing that N-Myc overexpression resulted in castrate-resistant tumors with NEPC or adenocarcinoma histology foci. These complementary results solidify N-Myc as a driver of the NEPC phenotype. In both studies, N-Myc was shown to form a complex with Aurora kinase A (Aurora-A) that stabilized N-Myc and that was sensitive to allosteric inhibitors to Aurora-A. These results showed the feasibility of exploiting the mutual dependence of N-Myc and Aurora-A to revert their oncogenic functions.

Although N-Myc is overexpressed in the majority of NEPC cases, there is a spectrum of N-Myc expression in CRPC samples with $20 \%$ of CRPC tumors demonstrating transcript levels in the range of NEPC. ${ }^{45,129}$ N-Myc overexpression in prostate adenocarcinoma was sufficient to drive the NE phenotype. ${ }^{129}$ Specifically, RNA-seq analyses from multiple stable LNCaP or 22Rv1 cell populations overexpressing N-Myc showed that N-Myc overexpression resulted repression of AR signaling and activation of PRC2 signaling and gene expression signatures associated with RB1 loss, ${ }^{129}$ all of which are molecular features associated with NEPC. ${ }^{45,50,127}$ These results suggest that $\mathrm{N}-\mathrm{Myc}$ is at least one driver of NE plasticity in PCA. Recent pre-clinical data also suggest that lineage plasticity can be observed in the context of RB1/TP53 loss. ${ }^{130,131}$ EZH2 inhibitors can also sensitizes NEPC tumor cells to enzalutamide. This suggests that reversing or delaying lineage plasticity with this type of epigenetic approach may provide a clinical benefit to a larger number of patients than previously appreciated and would extend clinical responses to antiandrogen therapy in the case of PCA. 


\section{Heterogeneity}

The Impact of Multifocality and Heterogeneity on Tracking Lethal CRPC

At radical prostatectomy (RP), $\sim 80 \%$ of patients harbor multiclonal (also referred to as multifocal) PCA, where spatially distinct tumor foci, which may show similar morphology and/or grade (Gleason score), are present in the same prostate. ${ }^{132,133}$ Multifocal PCA represents clones of independent origin, as supported by numerous approaches, including ERG rearrangement status (by FISH or IHC); ${ }^{27,134-145}$ in contrast, lethal, metastatic CRPC appears uniformly $E R G$ rearrangement positive or negative in all sites in a given patient, consistent with clonal origin, although extensive subclonal structure is present. 47,95,96,98,99,146,147

$E R G$ rearrangement status $\left(\mathrm{ERG}^{+}\right.$or $\left.\mathrm{ERG}^{-}\right)$is a useful clonal marker to demonstrate spatially distant multifocal tumors.135,137,141,142,144,145,148 Several anecdotal NGS studies ( $n$-values $\leq 5-10$ ) have added complexity to tracking the eventual CRPC clone through identifying intrafocal heterogeneity at RP. 95,98,99,149-152 These series of locally advanced PCA vary from reporting little divergence to complete lack of shared alterations between the index focus and lymph node metastases and/or CRPC. Haffner et $a l^{73}$ tracked the lethal clone in a single patient. Remarkably, they found that at RP, a small, organ confined low-grade (Gleason score 6) area of a large, high-grade primary tumor was the only area that harbored all alterations present in distant CRPC and lethal metastases. Critically, these alterations were absent from the vast majority of the primary tumor and lymph node metastasis at RP. Hence, in this patient, the lethal CRPC clone arose from a small, low-grade area of a histologically defined single index focus, rather than the higher grade area or concurrent lymph node metastasis. Whether this ' $n$ of 1' case represents the exception, rather than the rule, can only be assessed in a large cohort of paired $\mathrm{RP}$ and CRPC specimens, rather than locally advanced PCA.

Gundem et $a l^{80}$ recently explored PCA clonal evolution in 10 men with heavily treated CRPC at rapid autopsy. Like other published rapid autopsy series, this cohort did not represent a clinical trial and did not include patients treated with current second line agents targeting AR signaling (enzalutamide and abiraterone). With these caveats, their study presents a key snapshot of heavily treated lethal CRPC. In their study, primary prostate tumors (retained during treatment of advanced disease) demonstrated the presence of a large 'trunk' of mutations seen subclonally. Among the mutations found in the trunk, a subset of potential driver mutations was observed in a more pure, clonal form in the metastatic lesions. They demonstrate the feasibility of tracking clonal mutations in metastases back to initiating lesions. Currently on-going studies from the SU2C-CRPC500 cohort are actively addressing this in a more formal manner.

In summary, the advent of the radical prostatectomy coupled with advances in genomics led for steady increase in our understanding of PCA genomics. More recently, the examination of patients treated for advanced CRPC either at time of autopsy or through metastatic biopsies is leading to important insights into tumor plasticity and resistance. Future advances in cancer mouse models and organoids derived from patients will enable the development of new treatment strategies.

\section{Disclosure/conflict of interest}

The authors declare no conflict of interest.

\section{References}

1 Mullins JK, Feng Z, Trock BJ, et al. The impact of anatomical radical retropubic prostatectomy on cancer control: the 30-year anniversary. J Urol 2012;188: 2219-2224.

2 Gao D, Vela I, Sboner A, et al. Organoid cultures derived from patients with advanced prostate cancer. Cell 2014;159:176-187.

3 Robinson D, Van Allen EM, Wu YM, et al. Integrative clinical genomics of advanced prostate cancer. Cell 2015;161:1215-1228.

4 Atkin NB, Baker MC. Chromosome 10 deletion in carcinoma of the prostate. N Engl J Med 1985;312:315.

5 Gibas Z, Becher R, Kawinski E, et al. A highresolution study of chromosome changes in a human prostatic carcinoma cell line (LNCaP). Cancer Genet Cytogenet 1984;11:399-404.

6 Arps S, Rodewald A, Schmalenberger B, et al. Cytogenetic survey of 32 cancers of the prostate. Cancer Genet Cytogenet 1993;66:93-99.

7 Atkin NB, Baker MC. Chromosome study of five cancers of the prostate. Hum Genet 1985;70:359-364.

8 Lundgren R, Mandahl N, Heim S, et al. Cytogenetic analysis of 57 primary prostatic adenocarcinomas. Genes Chromosomes Cancer 1992;4:16-24.

9 Baker SJ, Fearon ER, Nigro JM, et al. Chromosome 17 deletions and p53 gene mutations in colorectal carcinomas. Science 1989;244:217-221.

10 Nigro JM, Baker SJ, Preisinger AC, et al. Mutations in the p53 gene occur in diverse human tumour types. Nature 1989;342:705-708.

11 Takahashi T, Nau MM, Chiba I, et al. p53: a frequent target for genetic abnormalities in lung cancer. Science 1989;246:491-494.

12 Carter BS, Ewing CM, Ward WS, et al. Allelic loss of chromosomes $16 \mathrm{q}$ and $10 \mathrm{q}$ in human prostate cancer. Proc Natl Acad Sci USA 1990;87:8751-8755.

13 Isaacs WB, Carter BS, Ewing CM. Wild-type p53 suppresses growth of human prostate cancer cells containing mutant p53 alleles. Cancer Res 1991;51: 4716-4720.

14 Macoska JA, Powell IJ, Sakr W, et al. Loss of the 17p chromosomal region in a metastatic carcinoma of the prostate. J Urol 1992;147:1142-1146. 
15 Navone NM, Troncoso P, Pisters LL, et al. p53 protein accumulation and gene mutation in the progression of human prostate carcinoma. J Natl Cancer Inst 1993;85:1657-1669.

16 Yunis JJ, Ramsay N. Retinoblastoma and subband deletion of chromosome 13. Am J Dis Child 1978;132: 161-163.

17 Friend SH, Bernards R, Rogelj S, et al. A human DNA segment with properties of the gene that predisposes to retinoblastoma and osteosarcoma. Nature 1986;323: 643-646.

18 Bookstein R, Shew JY, Chen PL, et al. Suppression of tumorigenicity of human prostate carcinoma cells by replacing a mutated RB gene. Science 1990;247: 712-715.

19 Bookstein R, Rio P, Madreperla SA, et al. Promoter deletion and loss of retinoblastoma gene expression in human prostate carcinoma. Proc Natl Acad Sci USA 1990;87:7762-7766.

20 Brooks JD, Bova GS, Isaacs WB. Allelic loss of the retinoblastoma gene in primary human prostatic adenocarcinomas. Prostate 1995;26:35-39.

21 Phillips SM, Barton CM, Lee SJ, et al. Loss of the retinoblastoma susceptibility gene (RB1) is a frequent and early event in prostatic tumorigenesis. Br J Cancer 1994;70:1252-1257.

22 Konig JJ, Kamst E, Hagemeijer A, et al. Cytogenetic characterization of several androgen responsive and unresponsive sublines of the human prostatic carcinoma cell line LNCaP. Urol Res 1989;17:79-86.

23 Visakorpi T, Kallioniemi AH, Syvanen AC, et al. Genetic changes in primary and recurrent prostate cancer by comparative genomic hybridization. Cancer Res 1995;55:342-347.

24 Suzuki H, Emi M, Komiya A, et al. Localization of a tumor suppressor gene associated with progression of human prostate cancer within a $1.2 \mathrm{Mb}$ region of 8p22-p21.3. Genes Chromosomes Cancer 1995;13: $168-174$.

25 Emmert-Buck MR, Vocke CD, Pozzatti RO, et al. Allelic loss on chromosome 8p12-21 in microdissected prostatic intraepithelial neoplasia. Cancer Res 1995;55:2959-2962.

26 Trapman J, Sleddens HF, van der Weiden MM, et al. Loss of heterozygosity of chromosome 8 microsatellite loci implicates a candidate tumor suppressor gene between the loci D8S87 and D8S133 in human prostate cancer. Cancer Res 1994;54:6061-6064.

27 Sakr WA, Macoska JA, Benson P, et al. Allelic loss in locally metastatic, multisampled prostate cancer. Cancer Res 1994;54:3273-3277.

28 Matsuyama H, Pan Y, Skoog L, et al. Deletion mapping of chromosome $8 \mathrm{p}$ in prostate cancer by fluorescence in situ hybridization. Oncogene 1994;9: 3071-3076.

29 Massenkeil G, Oberhuber H, Hailemariam S, et al. P53 mutations and loss of heterozygosity on chromosomes $8 p, 16 q, 17 p$, and $18 q$ are confined to advanced prostate cancer. Anticancer Res 1994;14:2785-2790.

30 Macoska JA, Trybus TM, Sakr WA, et al. Fluorescence in situ hybridization analysis of $8 p$ allelic loss and chromosome 8 instability in human prostate cancer. Cancer Res 1994;54:3824-3830.

31 MacGrogan D, Levy A, Bostwick D, et al. Loss of chromosome arm 8p loci in prostate cancer: mapping by quantitative allelic imbalance. Genes Chromosomes Cancer 1994;10:151-159.
32 Cher ML, MacGrogan D, Bookstein R, et al. Comparative genomic hybridization, allelic imbalance, and fluorescence in situ hybridization on chromosome 8 in prostate cancer. Genes Chromosomes Cancer 1994;11:153-162.

33 Bova GS, Carter BS, Bussemakers MJ, et al. Homozygous deletion and frequent allelic loss of chromosome 8p22 loci in human prostate cancer. Cancer Res 1993;53:3869-3873.

34 Bergerheim US, Kunimi K, Collins VP, et al. Deletion mapping of chromosomes 8, 10, and 16 in human prostatic carcinoma. Genes Chromosomes Cancer 1991;3:215-220.

35 Bova GS, MacGrogan D, Levy A, et al. Physical mapping of chromosome 8p22 markers and their homozygous deletion in a metastatic prostate cancer. Genomics 1996;35:46-54.

36 He WW, Sciavolino PJ, Wing J, et al. A novel human prostate-specific, androgen-regulated homeobox gene (NKX3.1) that maps to $8 \mathrm{p} 21$, a region frequently deleted in prostate cancer. Genomics 1997;43:69-77.

37 Hughes IA, Davies JD, Bunch TI, et al. Androgen insensitivity syndrome. Lancet 2012;380:1419-1428.

38 Shukla GC, Plaga AR, Shankar E, et al. Androgen receptor-related diseases: what do we know? Andrology 2016;4:366-381.

39 Veldscholte J, Ris-Stalpers C, Kuiper GG, et al. A mutation in the ligand binding domain of the androgen receptor of human LNCaP cells affects steroid binding characteristics and response to antiandrogens. Biochem Biophys Res Commun 1990;173: 534-540.

40 Newmark JR, Hardy DO, Tonb DC, et al. Androgen receptor gene mutations in human prostate cancer. Proc Natl Acad Sci USA 1992;89:6319-6323.

41 Taplin ME, Bubley GJ, Shuster TD, et al. Mutation of the androgen-receptor gene in metastatic androgenindependent prostate cancer. N Engl J Med 1995;332: 1393-1398.

42 Visakorpi T, Hyytinen E, Koivisto $\mathrm{P}$, et al. In vivo amplification of the androgen receptor gene and progression of human prostate cancer. Nat Genet 1995;9:401-406.

43 Bubendorf L, Kononen J, Koivisto P, et al. Survey of gene amplifications during prostate cancer progression by high-throughout fluorescence in situ hybridization on tissue microarrays. Cancer Res 1999;59: 803-806.

44 Cancer Genome Atlas Research N. The molecular taxonomy of primary prostate cancer. Cell 2015;163: 1011-1025.

45 Beltran H, Prandi D, Mosquera JM, et al. Divergent clonal evolution of castration-resistant neuroendocrine prostate cancer. Nat Med 2016;22:298-305.

46 Beltran H, Yelensky R, Frampton GM, et al. Targeted next-generation sequencing of advanced prostate cancer identifies potential therapeutic targets and disease heterogeneity. Eur Urol 2013;63:920-926.

47 Grasso CS, Wu YM, Robinson DR, et al. The mutational landscape of lethal castration-resistant prostate cancer. Nature 2012;487:239-243.

48 Kumar A, Coleman I, Morrissey C, et al. Substantial interindividual and limited intraindividual genomic diversity among tumors from men with metastatic prostate cancer. Nat Med 2016;22:369-378.

49 Sun S, Sprenger CC, Vessella RL, et al. Castration resistance in human prostate cancer is conferred by a 
frequently occurring androgen receptor splice variant. J Clin Invest 2010;120:2715-2730.

50 Beltran H, Rickman DS, Park K, et al. Molecular characterization of neuroendocrine prostate cancer and identification of new drug targets. Cancer Discov 2011;1:487-495.

51 Gray IC, Phillips SM, Lee SJ, et al. Loss of the chromosomal region 10q23-25 in prostate cancer. Cancer Res 1995;55:4800-4803.

$52 \mathrm{Li} \mathrm{J}$, Yen C, Liaw D, et al. PTEN, a putative protein tyrosine phosphatase gene mutated in human brain, breast, and prostate cancer. Science 1997;275: 1943-1947.

53 Rubin MA, Gerstein A, Reid K, et al. 10q23.3 loss of heterozygosity is higher in lymph node-positive (pT2$3, \mathrm{N+}$ ) versus lymph node-negative (pT2-3,N0) prostate cancer. Hum Pathol 2000;31:504-508.

54 Fleming WH, Hamel A, MacDonald R, et al. Expression of the c-myc protooncogene in human prostatic carcinoma and benign prostatic hyperplasia. Cancer Res 1986;46:1535-1538.

55 Jenkins RB, Qian J, Lieber MM, et al. Detection of c-myc oncogene amplification and chromosomal anomalies in metastatic prostatic carcinoma by fluorescence in situ hybridization. Cancer Res 1997;57:524-531.

56 Clegg NJ, Couto SS, Wongvipat J, et al. MYC cooperates with AKT in prostate tumorigenesis and alters sensitivity to mTOR inhibitors. PLoS ONE 2011;6:e17449.

57 Ellwood-Yen K, Graeber TG, Wongvipat J, et al. Mycdriven murine prostate cancer shares molecular features with human prostate tumors. Cancer Cell 2003;4:223-238

58 Lapointe J, Li C, Giacomini CP, et al. Genomic profiling reveals alternative genetic pathways of prostate tumorigenesis. Cancer Res 2007;67: 8504-8510.

59 Rubin MA, Girelli G, Demichelis F. Genomic correlates to the newly proposed grading prognostic groups for prostate cancer. Eur Urol 2016;69:557-560.

60 Hieronymus H, Schultz N, Gopalan A, et al. Copy number alteration burden predicts prostate cancer relapse. Proc Natl Acad Sci USA 2014;111: 11139-11144.

61 Tomlins SA, Rhodes DR, Perner S, et al. Recurrent fusion of TMPRSS2 and ETS transcription factor genes in prostate cancer. Science 2005;310: 644-648.

62 Berger MF, Lawrence MS, Demichelis F, et al. The genomic complexity of primary human prostate cancer. Nature 2011;470:214-220.

63 Baca SC, Prandi D, Lawrence MS, et al. Punctuated evolution of prostate cancer genomes. Cell 2013;153: 666-677.

64 Barbieri CE, Baca SC, Lawrence MS, et al. Exome sequencing identifies recurrent SPOP, FOXA1 and MED12 mutations in prostate cancer. Nat Genet 2012;44:685-689.

65 Tomlins SA, Mehra R, Rhodes DR, et al. TMPRSS2: ETV4 gene fusions define a third molecular subtype of prostate cancer. Cancer Res 2006;66:3396-3400.

66 Esgueva R, Perner S, JL C, et al. Prevalence of TMPRSS2-ERG and SLC45A3-ERG gene fusions in a large prostatectomy cohort. Mod Pathol 2010.

67 Han B, Mehra R, Dhanasekaran SM, et al. A fluorescence in situ hybridization screen for E26 transformation-specific aberrations: identification of DDX5-ETV4 fusion protein in prostate cancer. Cancer Res 2008;68:7629-7637.

68 Maher CA, Palanisamy N, Brenner JC, et al. Chimeric transcript discovery by paired-end transcriptome sequencing. Proc Natl Acad Sci USA 2009;106:12353-12358.

69 Pflueger D, Rickman DS, Sboner A, et al. N-myc downstream regulated gene 1 (NDRG1) is fused to ERG in prostate cancer. Neoplasia 2009;11:804-811.

70 Palanisamy N, Ateeq B, Kalyana-Sundaram S, et al. Rearrangements of the RAF kinase pathway in prostate cancer, gastric cancer and melanoma. Nat Med 2010;16:793-798.

71 Mani RS, Chinnaiyan AM. Triggers for genomic rearrangements: insights into genomic, cellular and environmental influences. Nat Rev Genet 2010;11:819-829.

72 Ju BG, Lunyak VV, Perissi V, et al. A topoisomerase IIbeta-mediated dsDNA break required for regulated transcription. Science 2006;312:1798-1802.

73 Haffner MC, Aryee MJ, Toubaji A, et al. Androgeninduced TOP2B-mediated double-strand breaks and prostate cancer gene rearrangements. Nat Genet 2010;42:668-675.

74 Bastus NC, Boyd LK, Mao X, et al. Androgen-induced TMPRSS2:ERG fusion in nonmalignant prostate epithelial cells. Cancer Res 2010;70:9544-9548.

75 Lin C, Yang L, Tanasa B, et al. Nuclear receptorinduced chromosomal proximity and DNA breaks underlie specific translocations in cancer. Cell 2009;139:1069-1083.

76 Mani RS, Tomlins SA, Callahan K, et al. Induced chromosomal proximity and gene fusions in prostate cancer. Science 2009;326:1230.

77 Cancer Genome Atlas Research Network. Electronic address scmo, Cancer Genome Atlas Research N. The molecular taxonomy of primary prostate cancer. Cell 2015;163:1011-1025.

78 Carter SL, Cibulskis K, Helman E, et al. Absolute quantification of somatic DNA alterations in human cancer. Nat Biotechnol 2012;30:413-421.

79 Prandi D, Baca SC, Romanel A, et al. Unraveling the clonal hierarchy of somatic genomic aberrations. Genome Biol 2014;15:439.

80 Gundem G, Van Loo P, Kremeyer B, et al. The evolutionary history of lethal metastatic prostate cancer. Nature 2015;520:353-357.

81 Stephens PJ, Greenman CD, Fu B, et al. Massive genomic rearrangement acquired in a single catastrophic event during cancer development. Cell 2011;144:27-40.

82 Fraser M, Sabelnykova VY, Yamaguchi TN, et al. Genomic hallmarks of localized, non-indolent prostate cancer. Nature 2017;541:359-364.

83 Boysen G, Barbieri CE, Prandi D, et al. SPOP mutation leads to genomic instability in prostate cancer. eLife 2015;4:e09207; doi: https//doi.org/10.7554/eLife.09207.

84 Barbieri CE, Baca SC, Lawrence MS, et al. Exome sequencing identifies recurrent SPOP, FOXA1 and MED12 mutations in prostate cancer. Nat Genet 2012;44:685-689.

85 Blattner M, Lee DJ, O'Reilly C, et al. SPOP mutations in prostate cancer across demographically diverse patient cohorts. Neoplasia 2014;16:14-20.

86 Theurillat JP, Udeshi ND, Errington WJ, et al. Prostate cancer. Ubiquitylome analysis identifies dysregulation of effector substrates in $S P O P$-mutant prostate cancer. Science 2014;346:85-89. 
87 Groner Anna C, Cato L, de Tribolet-Hardy J, et al. TRIM24 is an oncogenic transcriptional activator in prostate cancer. Cancer Cell 2016;29:846-858.

88 An J, Wang C, Deng Y, et al. Destruction of full-length androgen receptor by wild-type SPOP, but not prostate-cancer-associated mutants. Cell Rep 2014;6: 657-669.

89 Geng C, Rajapakshe K, Shah SS, et al. Androgen receptor is the key transcriptional mediator of the tumor suppressor SPOP in prostate cancer. Cancer Res 2014;74:5631-5643.

90 Pierce WK, Grace CR, Lee J, et al. Multiple weak linear motifs enhance recruitment and processivity in SPOP-mediated substrate ubiquitination. J Mol Biol 2015.

91 González-Billalabeitia E, Seitzer N, Song SJ, et al. Vulnerabilities of PTEN-p53-deficient prostate cancers to compound PARP/PI3K inhibition. Cancer Discov 2014;4:896-904.

92 Geng C, He B, Xu L, et al. Prostate cancer-associated mutations in speckle-type POZ protein $(S P O P)$ regulate steroid receptor coactivator 3 protein turnover. Proc Natl Acad Sci USA 2013;110:6997-7002.

93 Blattner M, Liu D, Robinson BD, et al. SPOP mutation drives prostate tumorigenesis in vivo through coordinate regulation of $\mathrm{PI} 3 \mathrm{~K} / \mathrm{mTOR}$ and $\mathrm{AR}$ signaling. Cancer Cell 2017;31:436-451.

94 Romanel A, Garritano S, Stringa B, et al. Inherited determinants of early recurrent somatic mutations in prostate cancer. Nat Commun 2017;8:48.

95 Aryee MJ, Liu W, Engelmann JC, et al. DNA methylation alterations exhibit intraindividual stability and interindividual heterogeneity in prostate cancer metastases. Sci Transl Med 2013;5169ra10.

96 Liu W, Laitinen S, Khan S, et al. Copy number analysis indicates monoclonal origin of lethal metastatic prostate cancer. Nat Med 2009;15:559-565.

97 Tomlins SA, Mehra R, Rhodes DR, et al. Integrative molecular concept modeling of prostate cancer progression. Nat Genet 2007;39:41-51.

98 Gundem G, Van Loo P, Kremeyer B, et al. The evolutionary history of lethal metastatic prostate cancer. Nature 2015;520:353-357.

99 Hong MK, Macintyre G, Wedge DC, et al. Tracking the origins and drivers of subclonal metastatic expansion in prostate cancer. Nat Commun 2015;6:6605.

100 Chen CD, Welsbie DS, Tran C, et al. Molecular determinants of resistance to antiandrogen therapy. Nat Med 2004;10:33-39.

101 Bastos DA, Dzik C, Rathkopf D, et al. Expanding androgen- and androgen receptor signaling-directed therapies for castration-resistant prostate cancer. Oncology (Williston Park) 2014;28:693-699.

102 Bluemn EG, Nelson PS. The androgen/androgen receptor axis in prostate cancer. Curr Opin Oncol 2012;24:251-257.

103 Chen Y, Sawyers CL, Scher HI. Targeting the androgen receptor pathway in prostate cancer. Curr Opin Pharmacol 2008;8:440-448.

104 Richter E, Srivastava S, Dobi A. Androgen receptor and prostate cancer. Prostate Cancer Prostatic Dis 2007;10:114-118.

105 Grossmann ME, Huang H, Tindall DJ. Androgen receptor signaling in androgen-refractory prostate cancer. J Natl Cancer Inst 2001;93:1687-1697.

106 Hovelson DH, McDaniel AS, Cani AK, et al. Development and validation of a scalable next-generation sequencing system for assessing relevant somatic variants in solid tumors. Neoplasia 2015;17: 385-399.

107 Kumar A, White TA, MacKenzie AP, et al. Exome sequencing identifies a spectrum of mutation frequencies in advanced and lethal prostate cancers. Proc Natl Acad Sci USA 2011;108:17087-17092.

108 Karantanos T, Corn PG, Thompson TC. Prostate cancer progression after androgen deprivation therapy: mechanisms of castrate resistance and novel therapeutic approaches. Oncogene 2013;32: 5501-5511.

109 Kypta RM, Waxman J. Wnt/beta-catenin signalling in prostate cancer. Nat Rev Urol 2012;9:418-428.

110 Rajan P, Sudbery IM, Villasevil ME, et al. Nextgeneration sequencing of advanced prostate cancer treated with androgen-deprivation therapy. Eur Urol 2014;66:32-39.

111 Carver BS, Chapinski C, Wongvipat J, et al. Reciprocal feedback regulation of PI3K and androgen receptor signaling in PTEN-deficient prostate cancer. Cancer Cell 2011;19:575-586.

112 Rickman DS, Beltran H, Demichelis F, et al. Biology and evolution of poorly differentiated neuroendocrine tumors. Nat Med 2017;23:1-10.

113 Lai SL, Brauch H, Knutsen T, et al. Molecular genetic characterization of neuroendocrine lung cancer cell lines. Anticancer Res 1995;15:225-232.

114 Mertz KD, Setlur SR, Dhanasekaran SM, et al. Molecular characterization of TMPRSS2-ERG gene fusion in the NCI-H660 prostate cancer cell line: a new perspective for an old model. Neoplasia 2007;9: 200-206.

115 Burchardt T, Burchardt M, Chen MW, et al. Transdifferentiation of prostate cancer cells to a neuroendocrine cell phenotype in vitro and in vivo. J Urol 1999;162:1800-1805.

116 Bang YJ, Pirnia F, Fang WG, et al. Terminal neuroendocrine differentiation of human prostate carcinoma cells in response to increased intracellular cyclic AMP. Proc Natl Acad Sci USA 1994;91: 5330-5334.

117 Qiu Y, Robinson D, Pretlow TG, et al. Etk/Bmx, a tyrosine kinase with a pleckstrin-homology domain, is an effector of phosphatidylinositol 3'-kinase and is involved in interleukin 6-induced neuroendocrine differentiation of prostate cancer cells. Proc Natl Acad Sci USA 1998;95:3644-3649.

118 Deng $\mathrm{X}$, Liu $\mathrm{H}$, Huang J, et al. Ionizing radiation induces prostate cancer neuroendocrine differentiation through interplay of CREB and ATF2: implications for disease progression. Cancer Res 2008;68: 9663-9670.

119 Lin D, Wyatt AW, Xue H, et al. High fidelity patientderived xenografts for accelerating prostate cancer discovery and drug development. Cancer Res 2014;74: 1272-1283.

120 True LD, Buhler K, Quinn J, et al. A neuroendocrine/ small cell prostate carcinoma xenograft-LuCaP 49. Am J Pathol 2002;161:705-715.

121 van Haaften-Day C, Raghavan D, Russell P, et al. Xenografted small cell undifferentiated cancer of prostate: possible common origin with prostatic adenocarcinoma. Prostate 1987;11:271-279.

122 Pinthus JH, Waks T, Schindler DG, et al. WISH-PC2: a unique xenograft model of human prostatic small cell carcinoma. Cancer Res 2000;60:6563-6567. 
123 Huijbers IJ, Bin Ali R, Pritchard C, et al. Rapid target gene validation in complex cancer mouse models using re-derived embryonic stem cells. EMBO Mol Med 2014;6:212-225.

124 Rudin CM, Poirier JT. MYC, MAX, and small cell lung cancer. Cancer Discov 2014;4:273-274.

125 Teicher BA. Targets in small cell lung cancer. Biochem Pharmacol 2014;87:211-219.

126 Wistuba II, Gazdar AF, Minna JD. Molecular genetics of small cell lung carcinoma. Semin Oncol 2001;28: $3-13$.

127 Lee JK, Phillips JW, Smith BA, et al. N-Myc drives neuroendocrine prostate cancer initiated from human prostate epithelial cells. Cancer Cell 2016;29:536-547.

128 Mosquera JM, Beltran H, Park K, et al. Concurrent AURKA and MYCN gene amplifications are harbingers of lethal treatment-related neuroendocrine prostate cancer. Neoplasia 2013;15:1-10.

129 Dardenne E, Beltran H, Benelli M, et al. N-Myc induces an EZH2-mediated transcriptional program driving neuroendocrine prostate cancer. Cancer Cell 2016;30:563-577.

$130 \mathrm{Ku}$ SY, Rosario S, Wang Y, et al. Rb1 and Trp53 cooperate to suppress prostate cancer lineage plasticity, metastasis, and antiandrogen resistance. Science 2017;355:78-83.

$131 \mathrm{Mu} \mathrm{P}$, Zhang Z, Benelli M, et al. SOX2 promotes lineage plasticity and antiandrogen resistance in TP53- and RB1-deficient prostate cancer. Science 2017;355:84-88.

132 Arora R, Koch MO, Eble JN, et al. Heterogeneity of Gleason grade in multifocal adenocarcinoma of the prostate. Cancer 2004;100:2362-2366.

133 Meiers I, Waters DJ, Bostwick DG. Preoperative prediction of multifocal prostate cancer and application of focal therapy: review 2007. Urology 2007;70:3-8.

134 Attard G, Jameson C, Moreira J, et al. Hormonesensitive prostate cancer: a case report of ETS gene fusion heterogeneity. J Clin Pathol 2008;62:373-376.

135 Barry M, Perner S, Demichelis F, et al. TMPRSS2-ERG fusion heterogeneity in multifocal prostate cancer: clinical and biologic implications. Urology 2007;70: 630-633.

136 Cheng L, Song SY, Pretlow TG, et al. Evidence of independent origin of multiple tumors from patients with prostate cancer. J Natl Cancer Inst 1998;90:233-237.

137 Furusato B, Gao CL, Ravindranath L, et al. Mapping of TMPRSS2-ERG fusions in the context of multi-focal prostate cancer. Mod Pathol 2008;21:67-75.

138 Kobayashi M, Ishida H, Shindo T, et al. Molecular analysis of multifocal prostate cancer by comparative genomic hybridization. Prostate 2008;68:1715-1724.

139 Kunju LP, Carskadon S, Siddiqui J, et al. Novel RNA hybridization method for the in situ detection of
ETV1, ETV4, and ETV5 gene fusions in prostate cancer. Appl Immunohistochem Mol Morphol 2014;22:e32-e40.

140 Lindberg J, Klevebring D, Liu W, et al. Exome sequencing of prostate cancer supports the hypothesis of independent tumour origins. Eur Urol 2013;63: 347-353.

141 Mehra R, Han B, Tomlins SA, et al. Heterogeneity of TMPRSS2 gene rearrangements in multifocal prostate adenocarcinoma: molecular evidence for an independent group of diseases. Cancer Res 2007;67:7991-7995.

142 Perner S, Svensson MA, Hossain RR, et al. ERG rearrangement metastasis patterns in locally advanced prostate cancer. Urology 2010;75:762-767.

143 Qian J, Bostwick DG, Takahashi S, et al. Chromosomal anomalies in prostatic intraepithelial neoplasia and carcinoma detected by fluorescence in situ hybridization. Cancer Res 1995;55:5408-5414.

144 Smith SC, Palanisamy N, Zuhlke KA, et al. HOXB13 G84E-related familial prostate cancers: a clinical, histologic, and molecular survey. Am J Surg Pathol 2014;38:615-626.

145 Young A, Palanisamy N, Siddiqui J, et al. Correlation of Urine TMPRSS2:ERG and PCA3 to ERG+ and Total Prostate Cancer Burden. Am J Clin Pathol 2012;138: 685-696.

146 Nickerson ML, Im KM, Misner KJ, et al. Somatic alterations contributing to metastasis of a castrationresistant prostate cancer. Hum Mutat 2013;34: 1231-1241.

147 Mehra R, Tomlins SA, Yu J, et al. Characterization of TMPRSS2-ETS gene aberrations in androgenindependent metastatic prostate cancer. Cancer Res 2008;68:3584-3590.

148 Furusato B, Tan SH, Young D, et al. ERG oncoprotein expression in prostate cancer: clonal progression of ERG-positive tumor cells and potential for ERG-based stratification. Prostate Cancer Prostatic Dis 2010;13: 228-237.

149 Brocks D, Assenov Y, Minner S, et al. Intratumor DNA methylation heterogeneity reflects clonal evolution in aggressive prostate cancer. Cell Rep 2014;8: 798-806.

150 VanderWeele DJ, Brown CD, Taxy JB, et al. Low-grade prostate cancer diverges early from high grade and metastatic disease. Cancer Sci 2014;105:1079-1085.

151 Lindberg J, Kristiansen A, Wiklund P, et al. Tracking the origin of metastatic prostate Ccancer. Eur Urol 2015;67:819-822.

152 Cooper CS, Eeles R, Wedge DC, et al. Analysis of the genetic phylogeny of multifocal prostate cancer identifies multiple independent clonal expansions in neoplastic and morphologically normal prostate tissue. Nat Genet 2015;47:367-372. 